\title{
Alpha Oscillations in the Human Brain Implement Distractor Suppression Independent of Target Selection
}

\author{
Malte Wöstmann, ${ }^{-M o h s e n ~ A l a v a s h, ~ a n d ~} \odot$ Jonas Obleser \\ Department of Psychology, University of Lübeck, Lübeck, Germany 23562
}

In principle, selective attention is the net result of target selection and distractor suppression. The way in which both mechanisms are implemented neurally has remained contested. Neural oscillatory power in the alpha frequency band $(\sim 10 \mathrm{~Hz})$ has been implicated in the selection of to-be-attended targets, but there is lack of empirical evidence for its involvement in the suppression of to-be-ignored distractors. Here, we use electroencephalography recordings of $N=33$ human participants (males and females) to test the preregistered hypothesis that alpha power directly relates to distractor suppression and thus operates independently from target selection. In an auditory spatial pitch discrimination task, we modulated the location (left vs right) of either a target or a distractor tone sequence, while fixing the other in the front. When the distractor was fixed in the front, alpha power relatively decreased contralaterally to the target and increased ipsilaterally. Most importantly, when the target was fixed in the front, alpha lateralization reversed in direction for the suppression of distractors on the left versus right. These data show that target-selection-independent alpha power modulation is involved in distractor suppression. Although both lateralized alpha responses for selection and for suppression proved reliable, they were uncorrelated and distractor-related alpha power emerged from more anterior, frontal cortical regions. Lending functional significance to suppression-related alpha oscillations, alpha lateralization at the individual, single-trial level was predictive of behavioral accuracy. These results fuel a renewed look at neurobiological accounts of selection-independent suppressive filtering in attention.

Key words: alpha oscillations; attention; auditory; selection; suppression

\section{Significance Statement}

Although well established models of attention rest on the assumption that irrelevant sensory information is filtered out, the neural implementation of such a filter mechanism is unclear. Using an auditory attention task that decouples target selection from distractor suppression, we demonstrate that two sign-reversed lateralized alpha responses reflect target selection versus distractor suppression. Critically, these alpha responses are reliable, independent of each other, and generated in more anterior, frontal regions for suppression versus selection. Prediction of single-trial task performance from alpha modulation after stimulus onset agrees with the view that alpha modulation bears direct functional relevance as a neural implementation of attention. Results demonstrate that the neurobiological foundation of attention implies a selection-independent alpha oscillatory mechanism to suppress distraction.

\section{Introduction}

Human goal-oriented behavior requires both, the selection of relevant target information and the suppression of irrelevant distraction. Although foundational theories of attention implied some form of distractor suppression (Broadbent, 1958; Treis-

\footnotetext{
Received Aug. 12, 2019; revised 0ct. 10, 2019; accepted 0ct. 11, 2019.

Author contributions: M.W., M.A., and J.0. designed research; M.W. and M.A. performed research; M.W., M.A., and J.O. analyzed data; M.W., M.A., and J.O. wrote the paper.

This work was supported by European Research Council Consolidator Grant AUDADAPT 646696 (to J.0.). We thank Elisabeth Ni and Franziska Scharata who helped acquire, manage, and archive the data.

The authors declare no competing financial interests.

Correspondence should be addressed to Malte Wöstmann at malte.woestmann@uni-luebeck.de or Jonas Obleser at jonas.obleser@uni-luebeck.de.

https://doi.org/10.1523/JNEUROSCl.1954-19.2019

Copyright $\odot 2019$ the authors
}

man, 1964), different neural implementations of suppression are conceivable. On the one hand, suppression might be contingent on selection, meaning that distractors outside the focus of attention are suppressed automatically (Noonan et al., 2018). On the other hand, distractor suppression might be an independent neurocognitive process (Aron, 2007) that adapts to changing characteristics of the distractor even in case the focus of attention is unchanged.

The power of brain oscillations in the alpha frequency band $(\sim 10 \mathrm{~Hz})$ robustly tracks when humans shift their focus of attention between sensory modalities (Adrian, 1944; Fu et al., 2001; de Pesters et al., 2016), to time points of anticipated target presentation (Rohenkohl and Nobre, 2011; Payne et al., 2013), or to a particular location in space (Worden et al., 2000; Sauseng et al., 
2005; Popov et al., 2019). Because alpha power drops in brain regions related to processing upcoming target stimuli (de Pesters et al., 2016), and because lower alpha power correlates with increased neural responses to the target (Gould et al., 2011; Wöstmann et al., 2019b) and enhanced behavioral measures of target detection (Thut et al., 2006), low alpha power is considered a signature of enhanced neural excitability to support target selection.

At the same time, alpha power does increase in brain regions that process distracting stimuli. Although high alpha power is considered a brain state of inhibited neural processing (Jensen and Mazaheri, 2010; Foxe and Snyder, 2011; Strauß et al., 2014), evidenced also by negative correlation of alpha power and brain activity measured in functional magnetic resonance imaging (Laufs et al., 2003), it is unclear at present whether high alpha power constitutes an independent signature of distractor suppression or a byproduct of target selection (Foster and Awh, 2018; Van Diepen et al., 2019).

To study the contribution of alpha oscillations to attentional selection and suppression, neuroscientists have used spatial cueing of an upcoming target location under competing distraction at another location. Across modalities, target cueing induces alpha power lateralization, that is, alpha power decreases in the hemisphere contralateral to the target and increases in the ipsilateral hemisphere (Haegens et al., 2011a; Bauer et al., 2012; Ahveninen et al., 2013). Alpha lateralization bears behavioral relevance: it is modulated stronger for correct than incorrect responses to the target stimulus (Haegens et al., 2011a; Wöstmann et al., 2016); and it modulates behavioral responses to the target if participants' endogenous alpha lateralization is stimulated transcranially (magnetically: Romei et al., 2010; or electrically: Wöstmann et al., 2018).

Critically, previous studies often confounded target and distractor location by design (Kelly et al., 2006; Wöstmann et al., 2016; van Diepen et al., 2016): whenever the target appeared on the left, the distractor was presented on the right, and vice versa. To unambiguously assign alpha lateralization to selection versus suppression, it is necessary to physically decouple target and distractor location during spatial attention. Addressing this, we here disentangled this conundrum by fixing the position of either an auditory target or distractor stimulus in the front of the listener. We then only varied the respective other stimulus to come from either the left or right side.

We find that lateralized alpha power is an autonomous (i.e., target-independent) signature of suppression that can track the location of the distractor. The lateralization of alpha power proves as a reliable neural signature, separating selection from suppression within individuals and in their underlying neural generators. Finally, the instantaneous degree of alpha lateralization after, but not before, stimulus onset predicts trial-by-trial variations in behavioral accuracy for detecting small pitch changes in the target sound.

\section{Materials and Methods}

Preregistration. Before data recording, we preregistered the study design, data sampling plan, hypotheses, and analyses procedures online with the Open Science Framework (https://osf.io/bv7zs). All data and analysis code will be made available upon reasonable request.

Participants. We analyzed data of $N=33$ right-handed participants $\left(\mathrm{M}_{\text {age }}=23.3\right.$ years; $\mathrm{SD}_{\text {age }}=3.9$ years; 22 females $)$. Data of three additional participants were recorded but excluded, because two of them had excessive electroencephalography (EEG) artifacts and one was unable to perform the task. Participants were financially compensated or received course credit. All procedures were approved by the local ethics committee of the University of Lübeck.
Auditory stimuli and task setup. Task design and stimuli were adapted from Dai et al. (2018). Because of a change in the stimulus sampling frequency, however, duration and pitch of auditory stimuli deviated slightly in the present study. The experiment was implemented in the Psychtoolbox (Brainard, 1997) for MATLAB, and conducted in a soundproof cabin. All auditory stimuli were presented at a sampling frequency of $48 \mathrm{kHz}$ at a comfortable level of $\sim 65 \mathrm{dBA}$.

On each trial, an auditory spatial cue $(10.9 \mathrm{kHz}$ low-pass filtered Gaussian noise; $0.46 \mathrm{~s}$ ) was presented at one location followed by two concurrent tone sequences presented at two different spatial locations (front, left, or right). Each tone sequence consisted of two $0.46 \mathrm{~s} \mathrm{complex}$ tones (fixed ISI of $46 \mathrm{~ms}$ ), one low-pitch tone and one high-pitch tone. All tones and the spatial cue were gated on and off with $92 \mathrm{~ms}$ cosine ramps.

The pitch of the low-pitch tone was fixed at $192.7 \mathrm{~Hz}$ (including 32 harmonics) for one sequence and at $300.4 \mathrm{~Hz}$ (including 2 harmonics) for the other sequence. Throughout the experiment, the fundamental frequency of the high-pitch tone in each sequence varied in semitones relative to the low-pitch tone using an adaptive tracking procedure (twoup-one-down) to arrive at $\sim 71 \%$ task accuracy (Levitt, 1971). Thus, after one incorrect response or two subsequent correct responses the pitch difference within each tone sequence was increased or decreased in steps of 0.05 semitones on the next trial, respectively. The initial pitch difference for the tracking procedure was obtained from a pre-experiment training session. The cue location (front vs side loudspeaker), the pitch direction within each sequence (increasing vs decreasing), and the assignment of tone sequences to the loudspeaker locations was balanced across trials and drawn randomly for an individual trial.

Tone sequences were presented in free field using a pair of loudspeakers (Logitech, X 140). The location of a speaker could be either front or side (i.e., 0 or $\pm 90^{\circ}$ azimuth relative to ear-nose-ear line). Loudspeakers were positioned at $\sim 70 \mathrm{~cm}$ distance to the participant's head. As the main experimental manipulation, the location of the side speaker changed between left and right across blocks of the experiment. To this end, the experimenter moved the side speaker accordingly in the breaks between blocks of the experiment. The other speaker was positioned in the front of the participant throughout. There were three blocks of the experiment with the side speaker positioned on the left side, and three blocks with the side speaker on the right. The order of blocks was counterbalanced across participants, and alternated between blocks with the side speaker on the left versus right. Within each block a participant completed 96 trials (each loudspeaker served as the target in 48 trials).

Experimental design. The present experimental design implemented four conditions. In experimental blocks with the distractor fixed in the front, the target was either on the left side (select-left condition) or on the right (select-right condition). In blocks with the target fixed in the front, the distractor was either on the left side (suppress-left condition) or on the right (suppress-right condition).

Procedure. At the start of each trial, after a jittered period of $\sim 1 \mathrm{~s}$ $(0.8-1.2 \mathrm{~s})$, an auditory spatial cue was presented on one loudspeaker to inform the participant about the target loudspeaker location. After a jittered period of $\sim 1.8 \mathrm{~s}$ (right-skewed distribution; median: $1.84 \mathrm{~s}$; truncated at 1.47 and $2.48 \mathrm{~s}$ ) relative to cue offset, two tone sequences were presented concurrently. Participants reported whether the tone sequence at the target location increased or decreased in pitch and how confident they were in this response using a response box with four buttons. Participants were instructed to fixate a cross in the middle of the response box throughout the experiment. Before the main experiment, a short training ensured that participants could perform the pitch discrimination task.

EEG recording and preprocessing. The EEG was recorded at 64 active scalp electrodes (Ag/Ag-Cl; ActiChamp, Brain Products) at a sampling rate of $1000 \mathrm{~Hz}$, with a DC-280 Hz bandwidth, against a left mastoid reference (channel TP9). All electrode impedances were kept below $\sim 30$ $\mathrm{k} \Omega$. To ensure equivalent placement of the EEG cap, the vertex electrode $(\mathrm{Cz})$ was placed at $50 \%$ of the distance between inion and nasion and between left and right ear lobes.

For EEG data analysis, we used the FieldTrip toolbox (Oostenveld et al., 2011) for MATLAB (R2013b/R2018a) and custom scripts. Offline, 
A EEG data of eye movement task projected through horizontal saccade-component

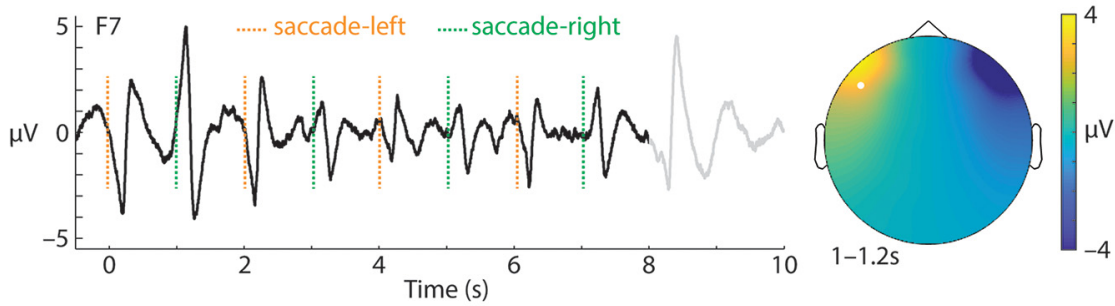

BEEG data of spatial attention task projected through horizontal saccade-component
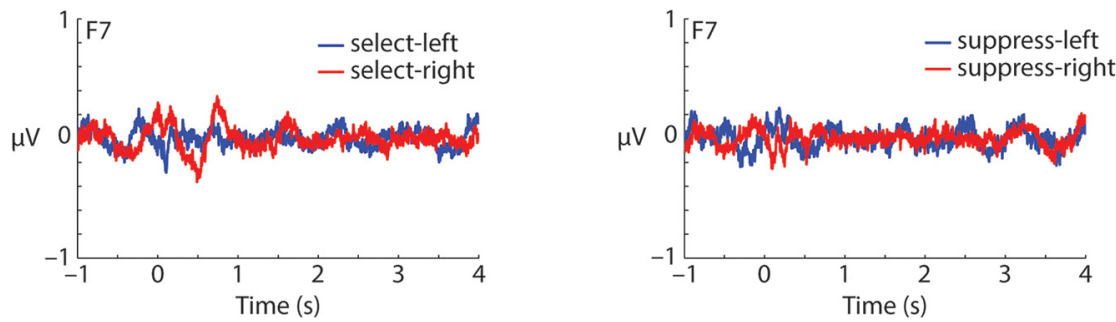

Figure 1. A, During a pre-experiment eye movement task participants followed a dot, which jumped eight times from the left to the right side on the screen, with their gaze. The grand-average ERP (at electrode F7) was computed on the EEG data projected through one horizontal saccade component per participant $(N=33)$. At $8 \mathrm{~s}$, participants performed an additional saccade back to the center of the screen to read task instructions. The topographic map shows ERP amplitude (1-1.2 s) following the onset of one saccade to the right side (electrode $F 7$, highlighted). $\boldsymbol{B}$, EEG data during the spatial attention task were projected through the same horizontal saccade component for each participant as in $\boldsymbol{A}$. Grand-average ERPs time locked to the onset of the auditory spatial cue show no obvious saccade-related activity in trials with targets or distractors on the left versus right side.

the continuous EEG data were filtered (1 Hz high-pass; $100 \mathrm{~Hz}$ low-pass) and segmented into epochs relative to the onset of the spatial cue ( -2 to $+6 \mathrm{~s}$ ). An independent component analysis (ICA) was used to detect and reject components corresponding to eye blinks, saccadic eye movements, muscle activity, and heartbeat. On average $38.48 \%$ (SD: 10\%) of components were rejected. After visual inspection of EEG time-domain data, noisy electrodes ( 1 electrode of 2 participants) were interpolated using the nearest neighbor approach implemented in FieldTrip. Finally, trials for which an individual EEG channel exceeded a range of $200 \mu \mathrm{V}$ were rejected. On average 568 trials (SD: 11 trials) of executed 576 trials per participant were used for further analyses.

Analysis of neural oscillatory activity. EEG data were re-referenced to the average of all electrodes and downsampled to $250 \mathrm{~Hz}$. Single-trial time-frequency representations were derived using complex Fourier coefficients for a moving time window (fixed length of $0.5 \mathrm{~s}$; Hanning taper; moving in steps of $0.04 \mathrm{~s}$ ) for frequencies $1-30 \mathrm{~Hz}$ with a resolution of $1 \mathrm{~Hz}$.

To quantify the impact of selection and suppression, single-trial power representations (squared magnitude of complex Fourier coefficients) were calculated for individual experimental conditions: select-left, selectright, suppress-left, and suppress-right. For each participant, two lateralization indices (LIs) were calculated on absolute oscillatory power (Pow). The first index quantifies oscillatory signatures of target selection:

$$
\mathrm{LI}_{\text {selection }}=\left(\mathrm{Pow}_{\text {select-left }}-\mathrm{Pow}_{\text {select-right }}\right) /\left(\mathrm{Pow}_{\text {select-left }}+\mathrm{Pow}_{\text {select-right }}\right) \text {. }
$$

Importantly, the second index quantifies oscillatory signatures of distractor suppression, which goes beyond what previous studies have analyzed:

$$
\mathrm{LI}_{\text {suppression }}=\left(\mathrm{Pow}_{\text {suppress-left }}-\mathrm{Pow}_{\text {suppress-right }}\right) /
$$

$$
\left(\text { Pow }_{\text {suppress-left }}+\text { Pow }_{\text {suppress-right }}\right) \text {. }
$$

For statistical analyses, we followed our preregistered analysis plan (https://osf.io/bv7zs). In brief, we averaged each LI across frequencies in the alpha band $(8-12 \mathrm{~Hz})$, the time interval from cue onset to earliest tone sequence onset $(0-1.9 \mathrm{~s})$, separately for two sets of 12 left and 12 right hemispheric occipito-parietal electrodes (TP9/10, TP7/8, CP5/6, $\mathrm{CP} 3 / 4, \mathrm{CP} 1 / 2, \mathrm{P} 7 / 8, \mathrm{P} 5 / 6, \mathrm{P} 3 / 4, \mathrm{P} 1 / 2, \mathrm{PO} 7 / 8, \mathrm{PO} 3 / 4$, and O1/2). For statistical comparisons of the LI (left vs right hemisphere; selection vs suppression), we used nonparametric permutation tests. The reported $p$ value corresponds to the relative number of absolute values of 10,000 dependent-samples $t$ statistics computed on data with permuted condition labels exceeding the absolute empirical $t$ value for the original data.

To determine reliability of lateralization indices, we divided each participant's trials into three consecutive portions (each consisting of $\sim 192$ trials, with 48 trials for each condition), followed by calculation of lateralization indices for each portion. Next, we calculated the reliability metric Cronbach's alpha (CA) for each lateralization index across the three portions. The $p$ value for CA was derived by the relative number of permuted CAs, derived from 10,000 permutations of single-subject lateralization indices within each one of the three portions, exceeding the empirical CA (Prelog et al., 2009).

For the nonsignificant Spearman correlation of the two lateralization indices $\left(\mathrm{LI}_{\text {selection }}\right.$ ? $\mathrm{LI}_{\text {suppression }}$ ) we report the Bayes factor (BF; computed for Kendall's tau in the software Jamovi). The BF indicates how many times more likely the observed data are under the alternative $\left(H_{1}\right)$ compared with the null hypothesis $\left(H_{0}\right)$. By convention, a $\mathrm{BF}>3$ begins to lend support to $H_{1}$, whereas a $\mathrm{BF}<0.33$ begins to lend support to $\mathrm{H}_{0}$ (Dienes, 2014).

Control for saccadic eye movements. Although participants were instructed to keep central gaze during the entire experiment, it might be that systematic differences in saccadic eye movements confounded the results (Quax et al., 2019), even in an auditory attention task. To rule this out, we inspected the EEG for independent components tuned to horizontal saccadic eye movements.

Before the main experiment, each participant performed a brief eye movement task. Participants followed a dot on the screen that jumped eight times either vertically (up and down by $\sim 8^{\circ}$ visual angle) or horizontally (left and right by $\sim 8^{\circ}$ visual angle) with inter-jump intervals of $1 \mathrm{~s}$. The task started with a horizontal movement trial and then alternated between 20 vertical and 20 horizontal movement trials.

Trials of the eye movement task were segmented into epochs relative to the onset of the first jump of the dot $(-1$ to $+10 \mathrm{~s})$. An ICA was used to extract one component for horizontal eye movements for each participant (vertical eye movements were not considered further in this study). The event-related potential (ERP) across all participant's horizontal eye movement components clearly differentiated between saccades to the left versus right side (Fig. 1A).

To control for potential confounds of horizontal saccadic eye movements in the EEG data of the spatial attention task, we projected each participant's raw task data (with no trials rejected) through the horizontal eye movement component, followed by computation of the ERP. For statistical analysis, we performed two cluster-based permutation tests to contrast the ERP during trials of the spatial attention task $(0-4 \mathrm{~s})$ for target selection on the left versus right side and distractor suppression on the left versus right side, respectively. In essence, these cluster-based permutation tests cluster $t$ values of adjacent bins in time-electrode space (minimum cluster size: 3 adjacent electrodes) and compare the summed $t$ statistic of the observed cluster against 10,000 randomly drawn clusters from the same data with permuted condition labels. The $p$ value of a cluster corresponds to the proportion of Monte Carlo iterations in which the summed $t$ statistic of the observed cluster is exceeded (two-sided testing; alpha level of 0.05).

ERPs of task data projected through the horizontal eye movement component did not show differences between experimental conditions (Fig. 1B). Cluster permutation tests revealed no significant differences in the ERP for selection of targets on the left versus right side (all cluster $p$ 
values $>0.19$ ) or suppression of distractors on the left versus right side (all cluster $p$ values $>$ $0.36)$.

In an additional control analysis, we also tested for gamma power $(>30 \mathrm{~Hz})$ differences in task data projected through horizontal eye movement components, which might be indicative of microsaccadic eye movements (Yuval-Greenberg et al., 2008). Two cluster permutation tests on time-frequency representations of oscillatory power in a broad frequency range $(1-90 \mathrm{~Hz})$ revealed no significant power differences for select-left versus selectright or suppress-left versus suppress-right conditions (all cluster $p$ values $>0.2$ ).

These results suggest that participants complied with our task instructions and did not systematically perform saccadic eye movements to or away from the to-be-selected or to-be-suppressed loudspeaker.

EEG source analysis. We used the Dynamic Imaging of Coherent Sources beamformer approach (Gross et al., 2001) implemented in FieldTrip. A standard head model (boundary element method; 3-shell) was used to calculate leadfields for a grid of $1 \mathrm{~cm}$ resolution. Spatial filters were calculated from the leadfield and the cross-spectral density of Fourier transforms centered at $10 \mathrm{~Hz}$ with $\pm 2 \mathrm{~Hz}$ spectral smoothing in the time interval $0-1.9 \mathrm{~s}$ relative to cue onset. For each participant, two spatial filters were calculated to source-localize $\mathrm{LI}_{\text {selection }}$ and $\mathrm{LI}_{\text {suppression }}$, based on all trials with the target or distractor on the side, respectively. The spatial filter for $\mathrm{LI}_{\text {selection }}$ was used to localize alpha power separately for select-left and select-right trials, followed by calculation of the lateralization index $\mathrm{LI}_{\text {selection }}$ on the source level (and accordingly for LI $_{\text {suppression }}$ ). Finally, source-level LIs were averaged across participants and mapped onto a standard brain surface.

Combined behavioral and EEG data analysis. Single-trial EEG and behavioral data were matched. Because of a few missing EEG triggers, one participant's data were excluded from behavioral analyses. For remaining $N=32$ participants, an average of 523 trials was used. Behavioral data were analyzed using mixed-effects models implemented in the fitlme function for MATLAB. The response variable of interest was single-trial confidence-weighted accuracy, derived by transformation of binary accuracy into 1 and $1 / 3$ for correct responses with respective high and low confidence, and into -1 and $-1 / 3$ for incorrect responses with respective high and low confidence (Wöstmann et al., 2015). As predictors, we used the titrated pitch difference (within both tone sequences), congruency of pitch direction across the two tone sequences (congruent versus incongruent), location of lateralized loudspeaker (left vs right), and role of lateralized loudspeaker (target vs distractor), with participant as a random intercept term, resulting in the linear-model expression: Confidence-weighted accuracy $\sim 1+$ Titrated pitch difference + Congruency of pitch direction $\times$ Location of lateralized loudspeaker $\times$ Role of lateralized loudspeaker $+(1 \mid$ Participant ID)

To model the relation of alpha lateralization and confidence-weighted accuracy, we included single-trial alpha lateralization $\left(\mathrm{LI}_{\text {single-trial }}\right)$ before tone sequence onset $(0-1.9 \mathrm{~s})$ and for a $1 \mathrm{~s}$ time window moving in $0.1 \mathrm{~s}$ steps through a trial as predictors in separate linear mixed-effects models. Single-trial alpha power lateralization was quantified as the contrast of alpha power (obtained via Fourier transform using multi-tapering at 10 $\mathrm{Hz}$ with $2 \mathrm{~Hz}$ spectral smoothing) at 12 parieto-occipital left-minus-right hemispheric electrodes:
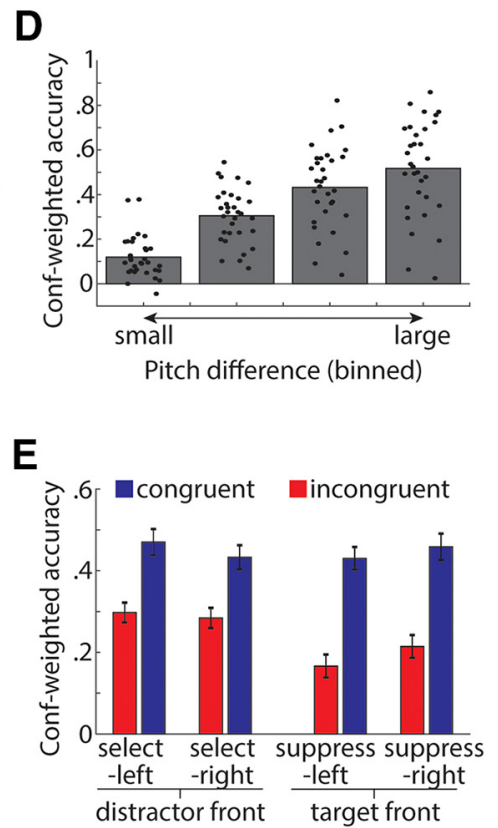

Figure 2. $\quad \boldsymbol{A}$, Trial design. Presentation of a broadband auditory cue $(0-10.9 \mathrm{kHz} ; 0.46 \mathrm{~s})$ was followed by a jittered silent (1.47-2.48 s). Next, two tone sequences, each consisting of two brief $(0.46 \mathrm{~s})$ complex tones, were presented at different Fz. Frequencies of high-frequency tones were titrated throughout the experiment. Participants had to judge whether the target 列 -0.5 to $0 \mathrm{~s}$; in decibel change), averaged across $N=33$ participants and all (64) scalp electrodes. $C$, Thin purple lines show 列 between the two tones within each tone sequence (divided into 4 bins for each participant for visualization), which was titrated of confidence-weighted accuracy, separately for congruent trials (both tone sequences increasing/decreasing in pitch; blue) and incongruent trials (one tone sequence increasing and other decreasing in pitch; red).

$\mathrm{LI}_{\text {single-trial }}=\left(\mathrm{Pow}_{\text {left-electrodes }}-\mathrm{Pow}_{\text {right-electrodes }}\right) /$

$$
\left(\text { Pow }_{\text {left-electrodes }}+\text { Pow }_{\text {right-electrodes }}\right) \text {. }
$$

\section{Results}

Participants $(N=33)$ performed a spatial pitch-discrimination task (Fig. 2A; adopted from Dai et al., 2018). The loudspeaker setup changed blockwise between front-and-left or front-andright, with one of the two loudspeakers serving as target and the other as distractor on each trial. A cue tone in the beginning of each trial indicated the location of the target loudspeaker. Participants had to report whether the ensuing tone sequence at the target location increased or decreased in pitch. A distracting tone sequence was presented simultaneously by the other loudspeaker.

As commonly observed in auditory attention tasks (Wöstmann et al., 2015, 2017a; Henry et al., 2017), power in the alpha frequency band $(8-12 \mathrm{~Hz})$ at parietal electrodes relatively increased after trial onset and decreased in the end of a trial before participants performed a behavioral response (Fig. $2 B, C$ ).

\section{Behavioral results}

To avoid ceiling and floor performance, the pitch difference within both tone sequences was titrated throughout the experiment (average pitch difference $=0.339$ semitones; betweensubject $\mathrm{SD}=0.524)$, using an adaptive procedure to target a proportion of $\sim 0.71$ correct responses. Average proportion correct was 0.715 (between-subject SD: 0.044) and average response time was $0.955 \mathrm{~s}$ (between-subject SD: 0.523). We modeled single- 
A

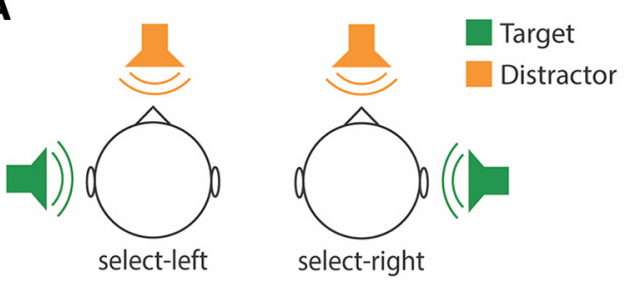

B
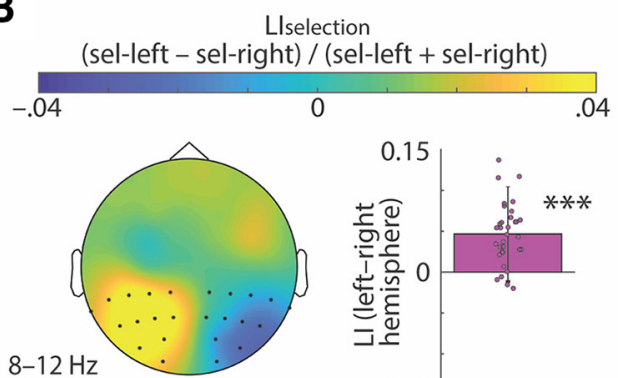

$0-1.9 \mathrm{~s}$

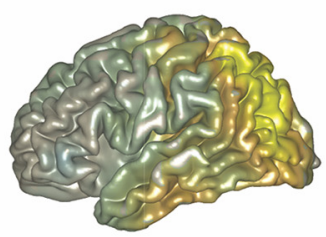

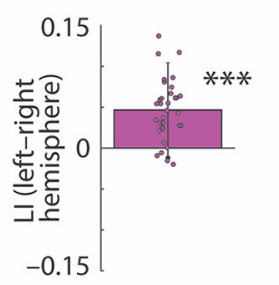

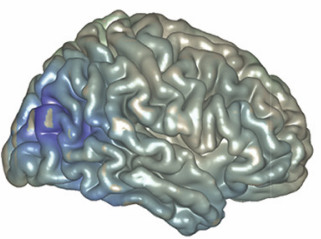

C

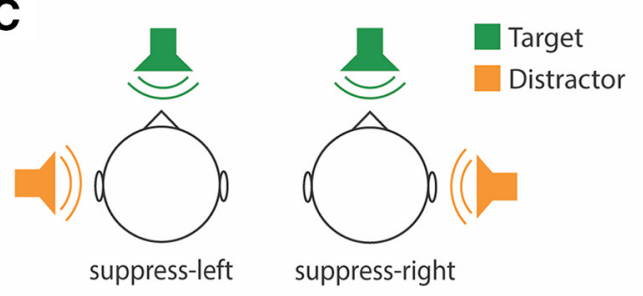

D \begin{tabular}{c} 
Llsuppression \\
(supp-left - supp-right) / (supp-left + supp-right) \\
-.02 \\
\multicolumn{2}{c}{0}
\end{tabular}
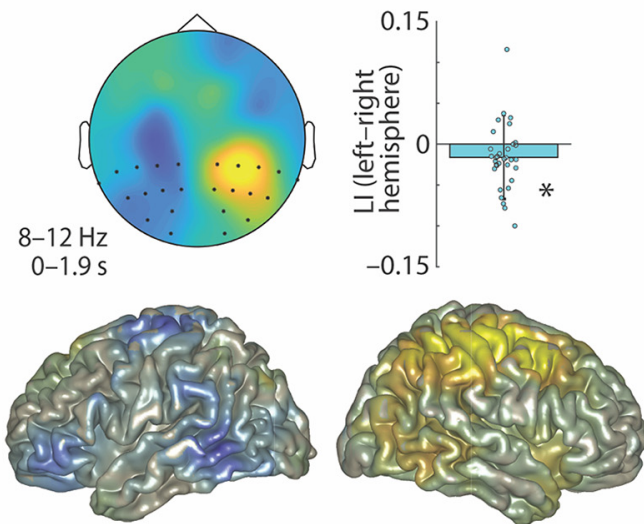

Figure 3. A, Schematic illustration of task setup in select-left and select-right trials. B, Topographic map and cortical surfaces show the lateralization index to contrast select-left and select-right trials under fixed distraction from the front $\left(\mathrm{LI}_{\text {selection }}\right)$. The $\mathrm{LI}$ was calculated for $8-12 \mathrm{~Hz}$ alpha power during anticipation of tone sequences $(0-1.9 \mathrm{~s})$. Bar graph, error bar, and dots show average, \pm 1 between-subject SEM, and single-subject differences of LI for highlighted parieto-occipital electrodes on the left versus right hemisphere, respectively. C, D, Same as $\boldsymbol{A}$ and $\boldsymbol{B}$, but for the LI for suppression of lateralized distractor stimuli $\left(\mathrm{LI}_{\text {suppression }}\right) .{ }^{*} p<0.05,{ }^{* * *} p<0.001$.

trial confidence-weighted accuracy on predictors titrated pitch difference (within both tone sequences), congruency of pitch direction across target and distractor tone sequence (congruent versus incongruent), location of lateralized loudspeaker (left versus right), and role of lateralized loudspeaker (target versus distractor).

Confidence-weighted accuracy increased if the pitch difference between the tones within each sequence (target and distractor) was larger (Fig. $2 D ; t_{(16949)}=10.031 ; p<0.001$ ), if tone sequences were congruent in pitch direction (Fig. $2 E ; t_{(16949)}=$ 8.572; $p<0.001$ ), and if the target loudspeaker was on the side compared with front (main effect role of lateralized loudspeaker: $t_{(16949)}=-5.622 ; p<0.001$ ), which agrees with a previous study that used a similar task design (Dai et al., 2018). The latter effect was driven by incongruent trials, evidenced by the congruency $X$ role of lateralized loudspeaker interaction $\left(t_{(16949)}=2.764 ; p=\right.$ $0.006)$. The location $\times$ role of lateralized loudspeaker interaction approached statistical significance $\left(t_{(16949)}=1.803 ; p=0.071\right)$, which speaks to a tendency of higher confidence-weighted accuracy for target selection on the left versus right but for distractor suppression on the right versus left.

\section{Alpha lateralization tracks target location independent of distractor}

We tested whether alpha power would track the spatial location of the to-be-selected target stimulus (left vs right) under fixed distraction from the front. To this end, we contrasted alpha power during the anticipation of tone sequences $(0-1.9 \mathrm{~s})$ for trials with a target on the left versus right side (Fig. $3 A, B$ ). Alpha power relatively increased in parietal, occipital, and inferior temporal regions in the hemisphere ipsilateral to the cued target lo- cation and decreased contralaterally. Accordingly, the $\mathrm{LI}_{\text {selection }}$ at left-minus-right parieto-occipital electrodes was significantly positive $\left[\mathrm{CI}_{95}=(0.033,0.06)\right.$; permutation $\left.p<0.001\right]$.

\section{Alpha lateralization tracks distractor location independent of target}

The most important objective of the present study was to test whether lateralized alpha power contains also a neural signature of distractor suppression, independent of target selection. To test this, we contrasted alpha power for trials with the target stimulus fixed in the front but with an anticipated distractor on the left versus right side (Fig. 3C,D). As predicted, the location of a tobe-suppressed distractor modulated alpha power orthogonally to target selection: alpha power relatively increased in parietal, posterior temporal, and frontal lobe regions in the hemisphere contralateral to the distractor and decreased ipsilaterally. Accordingly, the $\mathrm{LI}_{\text {suppression }}$ at parieto-occipital electrodes on the left versus right hemisphere was significantly negative $\left[\mathrm{CI}_{95}=\right.$ $(-0.03,-0.002)$; permutation $p=0.0202]$.

Having established EEG signatures of target selection $\left(\mathrm{LI}_{\text {selection }}\right)$ and distractor suppression ( $\mathrm{LI}_{\text {suppression }}$ ), we tested reliability of these signatures. Only if they are sufficiently reliable, meaning that results on repeated tests correlate positively, any relation or difference between the two signatures can be interpreted in a meaningful way. We divided each participant's data into three consecutive portions and found significant positive values of the reliability metric $\mathrm{CA}$ of alpha lateralization for target selection $\left(\mathrm{LI}_{\text {selection }} ; C A=0.602\right.$; permutation $\left.p=0.001\right)$ and distractor suppression $\left(\mathrm{LI}_{\text {suppression }} ; C A=0.522\right.$; permutation $\left.p=0.006\right)$. 
Alpha signatures of selection and suppression operate independently As for a potential hierarchy between alpha mechanisms of selection versus suppression, we tested whether anticipation of tobe-selected targets induces stronger alpha lateralization than anticipation of to-besuppressed distractors. The data speak clearly in favor of this hypothesis: the hemispheric difference in alpha lateralization (LI; Fig. 3, bar graphs) was significantly more positive for $\mathrm{LI}_{\text {selection }}$ than it was negative for $\mathrm{LI}_{\text {suppression }}\left[\mathrm{CI}_{95}=\right.$ $(0.008,0.053)$; permutation $p=0.005$ ]

We then asked, to what extent alpha lateralization for target selection and distractor suppression would relate. If the two neural signatures instantiate the same underlying cognitive faculty, participants with stronger alpha lateralization for target selection should show stronger alpha lateralization for distractor suppression as well. This would result in more positive $\mathrm{LI}_{\text {selection }}$ being accompanied with more negative $\mathrm{LI}_{\text {suppression }}$ and thus in a negative correlation. To the contrary, $\mathrm{LI}_{\text {selection }}$ and $\mathrm{LI}_{\text {suppression }}$ were not significantly correlated (Fig. 4A; $r_{\text {Spearman }}=0.153 ; p=$ 0.393 ; $\mathrm{BF}=0.294)$. Thus, alpha lateralization for target selection and distractor suppression can be considered two largely independent neural signatures.

Next, we tested whether alpha lateralization for target selection and distractor suppression might be implemented by different neural generators. To this end, we focused on differences in spatial distribution but not strength or direction of the hemispheric difference of alpha power modulation. We $z$-transformed each participant's lateralization indices, followed by taking their magnitudes ( $\mathrm{LI}_{\text {selection_norm }}$ and $\left.\mathrm{LI}_{\text {suppression_norm }}\right)$. Indeed, neural generators of selection and suppression differed significantly: target selection was driven by relatively stronger alpha power modulation in parieto-occipital cortex regions, primarily in the left hemisphere (Fig. $4 B$, pink regions). Conversely, neural generators of distractor suppression were modulated relatively stronger in more widespread, right superior and inferior parietal, inferior temporal, superior frontal, and middle frontal cortex regions (Fig. 4B, blue regions).

\section{Single-trial alpha lateralization predicts task performance}

To test our hypothesis that lateralized alpha power predicts task performance, we added single-trial alpha lateralization $\left(\mathrm{LI}_{\text {single- }}\right.$ trial) before the onset of tone sequences $(0-1.9 \mathrm{~s})$ to the set of predictors used in the analysis of behavioral results (Fig. 2D,E). Relatively higher left-than-right hemispheric alpha power should be beneficial in select-left and suppress-right trials but detrimental in select-right and suppress-left trials, reflected in a location $\times$ role of lateralized loudspeaker $\times$ alpha lateralization interaction. Against what we had hypothesized, this interaction was clearly nonsignificant $\left(t_{(16941)}=0.002 ; p=0.999\right)$. However, when we used single-trial alpha lateralization for successive time intervals throughout a trial as predictors (in separate mixed-effects models) in an exploratory follow-up analysis, we found a weak but significant location $\times$ role of lateralized loudspeaker $\times$ alpha lateralization interaction only in a late time window in the end of tone sequence presentation (3.1-3.4 s; Fig. 5A). The interaction was driven by trials with the lateralized loudspeaker on the right side (Fig. 5B): in line with the proposed inhibitory role of alpha power, relatively higher left-hemispheric alpha power improved suppression of distractors on the right but impaired selection of targets on the right.

\section{Discussion}

The present study tested whether the human brain implements a mechanism of distractor suppression that is independent of target selection. Results show that this is the case. Under fixed presentation of auditory targets in the front, anticipation of tobe-suppressed distractors on the left versus right side induced contralateral increase and ipsilateral decrease of alpha power. Alpha lateralization for target selection and distractor suppression were not only opposite in direction but we demonstrate here that the two are reliable and independent neural signatures generated in partly distinct underlying neural networks. Exploratory 

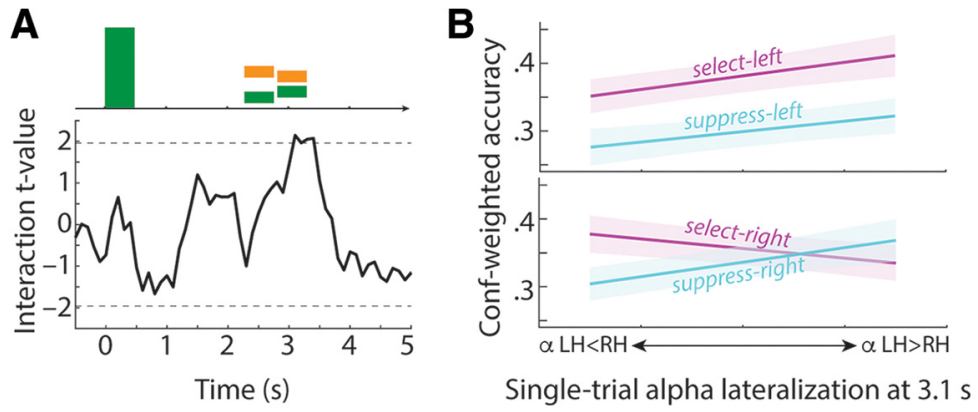

Figure 5. A, Black solid line shows trace of $t$ values for the location $\times$ role of lateralized loudspeaker $\times$ single-trial alpha lateralization interaction obtained from multiple linear mixed models. Dashed lines indicate significance thresholds (alpha $=$ 0.05; uncorrected). $\boldsymbol{B}$, Visualization of significant location $\times$ role of lateralized loudspeaker $\times$ alpha lateralization interaction at a latency of $3.1 \mathrm{~s}$. For each participant and experimental condition, single-trial alpha lateralization values ( $\mathrm{LI}_{\text {single-trial; quantifying }}$ left-vs-right hemispheric alpha power) were separated into four bins, followed by fitting a linear slope to average confidence (Conf)-weighted accuracy as a function of increasing bin number. Lines show average across single-subject linear fits, shaded areas show \pm 1 between-subject SEM. LH, left hemisphere; $\mathrm{RH}$, right hemisphere.

analysis of the relation between alpha lateralization and behavioral task performance supports the view that alpha power modulation following stimulus onset controls the readout of sensory objects that compete for attention.

\section{Alpha lateralization is an autonomous signature of} distractor suppression

The most important result of the present study was significant lateralization of alpha power in anticipation of distractors on the left versus right side in case the focus of attention was fixed to the front (Fig. 3C,D). This finding confirms our preregistered hypothesis of alpha lateralization as a neural signature of target-independent (i.e., autonomous) distractor suppression (https://osf.io/bv7zs).

Although neuroscience has previously shown that alpha oscillations represent an inhibitory signal that correlates with suppressed neural firing (Haegens et al., 2011b; Jensen et al., 2012), we demonstrate here that alpha power constitutes inhibition also in a psychological sense (Aron, 2007): it signifies a participant's intention to ignore distracting sensory information. Our results clearly challenge the view that alpha oscillations are not involved in distractor suppression (Foster and Awh, 2018) and instead show that lateralized alpha power adapts to the location of anticipated distraction independent of selecting a target at a fixed location.

Participants in the present study made use of alpha power lateralization, although in opposite direction, to implement target selection versus distractor suppression. In this sense, alpha power is a neural signal that implements the psychological construct of an attentional filter (Lakatos et al., 2013; Wöstmann et al., 2016) to coordinate the interplay of selection and suppression. Because alpha responses for selection and suppression were reliable and uncorrelated, we consider these neural responses traits that allow to place a participant's instantiation of the attentional filter in a two-dimensional space defined by neural target selection and distractor suppression (Fig. 4).

In theory, separation of competing target and distractor can be achieved by target enhancement or by distractor inhibition. So why should the human brain implement both of these mechanisms if one would suffice? For any biological system, the magnitude of response has a limited dynamic range. Attentional selection can only enhance neural processing of the target to a finite extent. Thus, a dual mechanism that additionally implements distractor suppression is able to effectively double the separation of target and distractor (Houghton and Tipper, 1994).
Neural implementation of target selection and distractor suppression

In line with the prevalent model of alpha power as a signature of neural inhibition (Jensen and Mazaheri, 2010; Foxe and Snyder, 2011; Strauß et al., 2014), relatively high alpha power in the hemisphere contralateral to a distractor indicates suppression, while low alpha power contralateral to a target indicates selection. Previous studies, which tied spatial locations of target and distractor by presenting either one left and the other right, likely observed a superposition of two underlying lateralized alpha responses that we separated in the present study.

Evidence for the involvement of alpha oscillations in neural processing of distraction comes also from studies that found alpha power modulation associated with changing features of the distractor, such as its acoustic quality (Wöstmann et al., 2017b), visual similarity to the target (Bonnefond and Jensen, 2012), the number of distractors (Sauseng et al., 2009), or continuous luminance changes in the distractor (Jia et al., 2019). The design of the present study allowed us to trace and contrast the neural sources of lateralized alpha responses for target selection and distractor suppression, which were prominent in superior and inferior parietal cortex region that are part of the dorsal attention network (Sadaghiani et al., 2010) and involved in coding spatial locations of stimuli in the environment (Colby and Goldberg, 1999).

The apparent absence of alpha lateralization in auditory cortex regions is not unusual for auditory spatial attention tasks in the EEG (Tune et al., 2018). Attentional modulation of alpha power in auditory cortex regions has been demonstrated mainly using brain imaging methods with high spatial specificity such as Magnetoencephalography (Wöstmann et al., 2016) or intracranial recordings (Gomez-Ramirez et al., 2011). Since the net EEG signal is dominated by widespread parieto-occipital alpha power, sophisticated analyses procedures such as the subtraction of visuallyand haptically-induced alpha modulation from auditory-induced alpha modulation (Spitzer et al., 2014) are necessary to reveal dominant sources of alpha modulation in auditory cortex regions. Furthermore, similar patterns of EEG alpha power lateralization in parietal cortex regions have been found for spatial shifts of visual but also auditory attention (Banerjee et al., 2011), suggesting that parietal alpha modulation is a signature of supramodal spatial attention. In this respect, it is likely that parietal alpha power lateralization in the present study reflects the allocation of supramodal attention rather than unimodal auditory attention alone.

Compared with target selection, distractor suppression induced relatively stronger alpha modulation in distributed regions including parietal, and (pre-) frontal cortex (Fig. 4). Such a pattern of frontal and parietal activations, termed multiple-demand system, has been found involved in a multitude of cognitively challenging tasks (Duncan, 2010). In particular, prefrontal cortex is a source of executive control (Miller and Cohen, 2001; Sadaghiani and Kleinschmidt, 2016), which is crucial for the orchestration of different neural processes to implement attention. Prefrontal cortex involvement in distractor suppression has also been evidenced by its relation to performance in tasks requiring cognitive control, such as the Stroop task (Vendrell et al., 1995). 
Our results thus help integrate the lateralized neural alpha response to distraction into models of prefrontal cortex involvement in inhibitory cognitive control.

It is of note that direct comparison of target selection and distractor suppression is somewhat limited in the present study. A bottom-up auditory cue drew attention to the target, whereas participants had to infer that the distractor would occur at the non-cued location. Furthermore, in theory, it might be possible that lateralized alpha oscillations code the relative position of the target with respect to the distractor, which was the same (i.e., $90^{\circ}$ to the left) in select-left and suppress-right trials. However, our results clearly speak against this, because alpha lateralization for target selection and distractor suppression were not only different in strength and source origin, but were also uncorrelated.

\section{Behavioral relevance of the lateralized alpha response}

Against what we had hypothesized, we did not find a significant relation of single-trial alpha lateralization before stimulus presentation and task performance (for a study that recently reported such a relation in a different task setting, see Dahl et al., 2019). In previous studies, we observed that the strength of the relation of alpha power modulation and behavior varied considerably between task settings, with some studies finding robust relations (Wöstmann et al., 2015, 2016), whereas others found weak (Wöstmann et al., 2019a) or no such relation (Wöstmann et al., 2017b).

In an exploratory follow-up analysis we found that alpha lateralization after the onset of competing tone sequences predicted single-trial pitch discrimination performance, although the relation was relatively weak. There is an ongoing debate whether alpha lateralization is a purely proactive mechanism of attentional control to prepare for upcoming target and distractor, or whether alpha lateralization also has the potency to reactively select the target and to suppresses the distractor after these have been encoded (for review of proactive and reactive mechanisms, see Geng, 2014).

Our results differentiate proactive and reactive accounts of attention: the observed alpha power lateralization before competing tone sequences speaks to alpha lateralization as a signature of proactive attentional control. However, prediction of pitch discrimination performance only by poststimulus alpha lateralization signifies the behavioral relevance of lateralized alpha power as a mechanism of reactive attentional control (Wöstmann et al., 2016).

\section{Conclusion}

Although well established models of attention rest on the assumption that irrelevant sensory information is filtered out, the neural implementation of such a filter mechanism is unclear. Using a task design that decouples target selection from distractor suppression, we demonstrate here that two independent lateralized alpha responses reflect target selection versus distractor suppression. These alpha responses are sign-reversed, reliable, and originate in more frontal, executive cortical regions for suppression than selection. Furthermore, lateralized alpha power predicts participants' accuracy in the judgment of a pitch change in the target stimulus. These findings support so-called "active suppression" models of attention, in which suppression is not a necessary byproduct of selection but an independent neurocognitive process.

\section{References}

Adrian ED (1944) Brain rhythms. Nature 153:360-362.

Ahveninen J, Huang S, Belliveau JW, Chang WT, Hämäläinen M (2013)
Dynamic oscillatory processes governing cued orienting and allocation of auditory attention. J Cogn Neurosci 25:1926-1943.

Aron AR (2007) The neural basis of inhibition in cognitive control. Neuroscientist 13:214-228.

Banerjee S, Snyder AC, Molholm S, Foxe JJ (2011) Oscillatory alpha-band mechanisms and the deployment of spatial attention to anticipated auditory and visual target locations: supramodal or sensory-specific control mechanisms? J Neurosci 31:9923-9932.

Bauer M, Kennett S, Driver J (2012) Attentional selection of location and modality in vision and touch modulates low-frequency activity in associated sensory cortices. J Neurophysiol 107:2342-2351.

Bonnefond M, Jensen O (2012) Alpha oscillations serve to protect working memory maintenance against anticipated distracters. Curr Biol 22:19691974.

Brainard DH (1997) The psychophysics toolbox. Spat Vis 10:433-436.

Broadbent D (1958) Perception and communication. London: Pergamon.

Colby CL, Goldberg ME (1999) Space and attention in parietal cortex. Annu Rev Neurosci 22:319-349.

Dahl MJ, Ilg L, Li SC, Passow S, Werkle-Bergner M (2019) Diminished pre-stimulus alpha-lateralization suggests compromised self-initiated attentional control of auditory processing in old age. Neuroimage 197:414424.

Dai L, Best V, Shinn-Cunningham BG (2018) Sensorineural hearing loss degrades behavioral and physiological measures of human spatial selective auditory attention. Proc Natl Acad Sci U S A 115:E3286-E3295.

de Pesters A, Coon WG, Brunner P, Gunduz A, Ritaccio AL, Brunet NM, Schalk G, de Weerd P, Roberts MJ, Oostenveld R, Fries P, Schalk G (2016) Alpha power indexes task-related networks on large and small scales: a multimodal ECoG study in humans and a non-human primate. Neuroimage 134:122-131.

Dienes Z (2014) Using Bayes to get the most out of non-significant results. Front Psychol 5:781.

Duncan J (2010) The multiple-demand (MD) system of the primate brain: mental programs for intelligent behaviour. Trends Cogn Sci 14:172-179.

Foster JJ, Awh E (2018) The role of alpha oscillations in spatial attention: limited evidence for a suppression account. Curr Opin Psychol 29:34-40.

Foxe JJ, Snyder AC (2011) The role of alpha-band brain oscillations as a sensory suppression mechanism during selective attention. Front Psychol 2:154.

Fu KM, Foxe JJ, Murray MM, Higgins BA, Javitt DC, Schroeder CE (2001) Attention-dependent suppression of distracter visual input can be crossmodally cued as indexed by anticipatory parieto-occipital alpha-band oscillations. Brain Res Cogn Brain Res 12:145-152.

Geng JJ (2014) Attentional mechanisms of distractor suppression. Curr Dir Psychol Sci 23:147-153.

Gomez-Ramirez M, Kelly SP, Molholm S, Sehatpour P, Schwartz TH, Foxe JJ (2011) Oscillatory sensory selection mechanisms during intersensory attention to rhythmic auditory and visual inputs: a human electrocorticographic investigation. J Neurosci 31:18556-18567.

Gould IC, Rushworth MF, Nobre AC (2011) Indexing the graded allocation of visuospatial attention using anticipatory alpha oscillations. J Neurophysiol 105:1318-1326.

Gross J, Kujala J, Hamalainen M, Timmermann L, Schnitzler A, Salmelin R (2001) Dynamic imaging of coherent sources: studying neural interactions in the human brain. Proc Natl Acad Sci U S A 98:694-699.

Haegens S, Händel BF, Jensen O (2011a) Top-down controlled alpha band activity in somatosensory areas determines behavioral performance in a discrimination task. J Neurosci 31:5197-5204.

Haegens S, Nácher V, Luna R, Romo R, Jensen O (2011b) $\alpha$-Oscillations in the monkey sensorimotor network influence discrimination performance by rhythmical inhibition of neuronal spiking. Proc Natl Acad Sci U S A 108:19377-19382.

Henry MJ, Herrmann B, Kunke D, Obleser J (2017) Aging affects the balance of neural entrainment and top-down neural modulation in the listening brain. Nat Commun 8:15801.

Houghton G, Tipper SP (1994) A model of inhibitory mechanisms in selective attention. In: Inhibitory processes of attention, memory and language (Dagenbach D, Carr T, eds). San Diego: Academic.

Jensen O, Mazaheri A (2010) Shaping functional architecture by oscillatory alpha activity: gating by inhibition. Front Hum Neurosci 4:186.

Jensen O, Bonnefond M, VanRullen R (2012) An oscillatory mechanism for prioritizing salient unattended stimuli. Trends Cogn Sci 16:200-206. 
Jia J, Fang F, Luo H (2019) Selective spatial attention involves two alphaband components associated with distinct spatiotemporal and functional characteristics. Neuroimage 199:228-236.

Kelly SP, Lalor EC, Reilly RB, Foxe JJ (2006) Increases in alpha oscillatory power reflect an active retinotopic mechanism for distracter suppression during sustained visuospatial attention. J Neurophysiol 95:3844-3851.

Lakatos P, Musacchia G, O'Connel MN, Falchier AY, Javitt DC, Schroeder CE (2013) The spectrotemporal filter mechanism of auditory selective attention. Neuron 77:750-761.

Laufs H, Kleinschmidt A, Beyerle A, Eger E, Salek-Haddadi A, Preibisch C, Krakow K (2003) EEG-correlated fMRI of human alpha activity. Neuroimage 19:1463-1476.

Levitt H (1971) Transformed up-down methods in psychoacoustics. J Acoust Soc Am 49:467.

Miller EK, Cohen JD (2001) An integrative theory of prefrontal cortex function. Annu Rev Neurosci 24:167-202.

Noonan MP, Crittenden BM, Jensen O, Stokes MG (2018) Selective inhibition of distracting input. Behav Brain Res 355:36-47.

Oostenveld R, Fries P, Maris E, Schoffelen JM (2011) FieldTrip: open source software for advanced analysis of MEG, EEG, and invasive electrophysiological data. Comput Intell Neurosci 2011:156869.

Payne L, Guillory S, Sekuler R (2013) Attention-modulated alpha-band oscillations protect against intrusion of irrelevant information. J Cogn Neurosci 25:1463-1476.

Popov T, Gips B, Kastner S, Jensen O (2019) Spatial specificity of alpha oscillations in the human visual system. Hum Brain Mapp 40:4432-4440.

Prelog AJ, Berry KJ, Mielke PW Jr (2009) Resampling permutation probability values for Cronbach's alpha. Percept Mot Skills 108:431-438.

Quax SC, Dijkstra N, van Staveren MJ, Bosch SE, van Gerven MAJ (2019) Eye movements explain decodability during perception and cued attention in MEG. Neuroimage 195:444-453.

Rohenkohl G, Nobre AC (2011) Alpha oscillations related to anticipatory attention follow temporal expectations. J Neurosci 31:14076-14084.

Romei V, Gross J, Thut G (2010) On the role of prestimulus alpha rhythms over occipito-parietal areas in visual input regulation: correlation or causation? J Neurosci 30:8692-8697.

Sadaghiani S, Kleinschmidt A (2016) Brain networks and alpha-oscillations: structural and functional foundations of cognitive control. Trends Cogn Sci 20:805-817.

Sadaghiani S, Scheeringa R, Lehongre K, Morillon B, Giraud AL, Kleinschmidt A (2010) Intrinsic connectivity networks, alpha oscillations, and tonic alertness: a simultaneous electroencephalography/functional magnetic resonance imaging study. J Neurosci 30:10243-10250.

Sauseng P, Klimesch W, Stadler W, Schabus M, Doppelmayr M, Hanslmayr S, Gruber WR, Birbaumer N (2005) A shift of visual spatial attention is selectively associated with human EEG alpha activity. Eur J Neurosci 22:2917-2926.

Sauseng P, Klimesch W, Heise KF, Gruber WR, Holz E, Karim AA, Glennon M, Gerloff C, Birbaumer N, Hummel FC (2009) Brain oscillatory substrates of visual short-term memory capacity. Curr Biol 19:1846-1852.
Spitzer B, Fleck S, Blankenburg F (2014) Parametric alpha- and beta-band signatures of supramodal numerosity information in human working memory. J Neurosci 34:4293-4302.

Strauß A, Wöstmann M, Obleser J (2014) Cortical alpha oscillations as a tool for auditory selective inhibition. Front Hum Neurosci 8:350.

Thut G, Nietzel A, Brandt SA, Pascual-Leone A (2006) Alpha-band electroencephalographic activity over occipital cortex indexes visuospatial attention bias and predicts visual target detection. J Neurosci 26:9494-9502.

Treisman AM (1964) Monitoring and storage of irrelevant messages in selective attention. J Verbal Learn Verbal Behav 3:449-459.

Tune S, Wöstmann M, Obleser J (2018) Probing the limits of alpha power lateralization as a neural marker of selective attention in middle-aged and older listeners. Eur J Neurosci 48:2537-2550.

Van Diepen RM, Foxe JJ, Mazaheri A (2019) The functional role of alphaband activity in attentional processing: the current zeitgeist and future outlook. Curr Opin Psychol 29:229-238.

van Diepen RM, Miller LM, Mazaheri A, Geng JJ (2016) The role of alpha activity in spatial and feature-based attention. eNeuro 3:ENEURO.020416.2016.

Vendrell P, Junqué C, Pujol J, Jurado MA, Molet J, Grafman J (1995) The role of prefrontal regions in the stroop task. Neuropsychologia 33:341-352.

Worden MS, Foxe JJ, Wang N, Simpson GV (2000) Anticipatory biasing of visuospatial attention indexed by retinotopically specific $\alpha$-band electroencephalography increases over occipital cortex. J Neurosci 20:RC63.

Wöstmann M, Herrmann B, Wilsch A, Obleser J (2015) Neural alpha dynamics in younger and older listeners reflect acoustic challenges and predictive benefits. J Neurosci 35:1458-1467.

Wöstmann M, Herrmann B, Maess B, Obleser J (2016) Spatiotemporal dynamics of auditory attention synchronize with speech. Proc Natl Acad Sci U S A 113:3873-3878.

Wöstmann M, Fiedler L, Obleser J (2017a) Tracking the signal, cracking the code: speech and speech comprehension in non-invasive human electrophysiology. Lang Cogn Neurosci 32:855-869.

Wöstmann M, Lim SJ, Obleser J (2017b) The human neural alpha response to speech is a proxy of attentional control. Cereb Cortex 27:3307-3317.

Wöstmann M, Vosskuhl J, Obleser J, Herrmann CS (2018) Opposite effects of lateralised transcranial alpha versus gamma stimulation on auditory spatial attention. Brain Stimul 11:752-758.

Wöstmann M, Schmitt LS, Obleser J (2019a) Does closing the eyes enhance auditory attention? Eye closure increases attentional alpha-power modulation but not listening performance. J Cognit Neurosci. Advance online publication. Retrieved March 26, 2019. doi:10.1162/jocn_a_01403.

Wöstmann M, Waschke L, Obleser J (2019b) Prestimulus neural alpha power predicts confidence in discriminating identical auditory stimuli. Eur J Neurosci 49:94-105.

Yuval-Greenberg S, Tomer O, Keren AS, Nelken I, Deouell LY (2008) Transient induced gamma-band response in EEG as a manifestation of miniature saccades. Neuron 58:429-441. 